\title{
Judicialización de las políticas sociales y cuestión social: subsidios para el debate
}

\author{
Juan Retana Jimenez*
}

\begin{abstract}
RESUMEN
El artículo destaca los elementos conceptuales que atraviesan el debate sobre la judicialización de la política, su significado y su interconexión con la política social. Es una cuestión reciente que viene aconteciendo desde mediados de la década de 1990 del siglo pasado en diversos países que sustentan Estados de Derecho concomitante a la entrada de políticas de corte neoliberal en la agenda pública. Se entiende que este debate atraviesa el Poder Judicial, la administración pública y las condiciones de vida, de resistencia y de acceso a las políticas sociales por parte de sujetos individuales y colectivos de la clase trabajadora. La línea de análisis es la de comprender la cuestión de la judicialización de las políticas sociales en sus contradicciones; considerándola como una estrategia de enfrentamiento que no supera la fragmentación de las demandas que surgen de las múltiples expresiones de la cuestión social por parte del Estado de Derecho contemporáneo.
\end{abstract}

PALABRAS CLAVES: Judicialización; política social; cuestión social.

\section{Judicialization of social policies and social issue: subsidies for debate}

\begin{abstract}
The article highlights the conceptual elements that are going through the debate on the judicialization of politics, its meaning and its interconnection with social policy. It is a recent issue that has been happening since the mid-1990s of the last century in various countries that support States of Law concomitant to the entry of neoliberal policies on the public agenda. It is understood that this debate is going through the Judiciary, public administration and living conditions, resistance and access to social policies by individual and collective subjects of the working class. The line of analysis is to understand the question of the judicialization of social policies in their contradictions; considering it as a confrontation strategy that does not overcome the fragmentation of the demands that arise from the multiple expressions of the social issue by the contemporary State of Law.
\end{abstract}

KEYWORDS: Judicialization; social policies; social issue.

Enviado em: 21/08/2019

Aprovado em: 20/10/2019

\section{Introducción}

La judicialización como cuestión contemporánea ha ganado notoriedad desde finales del siglo XX, se expande de diversas formas dentro de las reglas del juego de la democracia

\footnotetext{
${ }^{*}$ Doutor em Serviço Social, Professor da Universidade Federal Fluminense, Faculdade de Serviço Social. E-mail: jrjrjuffro@gmail.com.
} 
burguesa; a su vez, la judicialización de la política social, considerada como una parte del llamado proceso de judicialización de las relaciones sociales y de la política, se presenta con posibilidades y límites en los Estados de Derecho constituidos, donde se han desarrollado estrategias de corte neoliberal desde la década de 1990 del siglo pasado, que tienden a minimizar el papel de la seguridad social en esos Estados, abriendo camino a recortes al presupuesto y disminución del gasto público, que inciden en procesos de exclusión social de segmentos de la clase trabajadora en su acceso a las políticas y programas sociales.

De esta manera surgen movimientos en defensa de las instituciones y garantías sociales, que utilizándose de medios legales constituidos dentro del Estado, han generado demandas que atraviesan al Poder Judicial; ante lo cual este Poder es llamado a asumir posiciones que garanticen la legislación social vigente. Es un camino que deriva en que el Poder Ejecutivo así como otros entes autónomos públicos, se vean condicionados a cumplir su responsabilidad legal de efectuar e implementar la gestión de políticas y programas sociales en beneficio de segmentos de la clase trabajadora que, de forma individual y/o colectiva, ganaron en la justicia el reconocimiento de su derecho. En este sentido, el enfoque aquí explicitado se centra en la funcionalidad que este proceso cumple en la dinámica de la sociedad, como respuesta a demandas planteadas por actores sociales, representantes de la clase trabajadora, que conforman litigios en las cortes y salas de justicia para garantizar sus derechos sociales y de ciudadanía.

El propósito de este artículo es realizar un análisis inmanente del concepto de judicialización que subsidie el debate sobre la llamada judicialización de la política social y sus implicaciones. De esta forma es pertinente discutir ¿Qué se entiende por judicialización? ¿Cuáles son sus límites y rango de acción en la vida social? ¿Cómo la política social ha venido siendo judicializada y cuál es el sentido que asume como estrategia de intervención del Estado en las expresiones de la cuestión social en la actualidad? Son preguntas que surgen al observar esta dinámica social e institucional, las cuales sirven de guía para contribuir con el esclarecimiento de la concepción dominante sobre judicialización, por medio de una revisión crítica de la misma.

\section{Judicialización: elementos para su interpretación y crítica.}

El concepto de judicialización fue incorporado contemporáneamente en el mundo político y académico, según Nunes Junior (JUNIOR,2008:158) por los autores Neal Tate y Torbjorn Vallinder, que promovieron un encuentro en Bologna, Italia, en 1992, y de ese 
encuentro fue publicada en forma colectiva el libro "The global expansion of judicial power" en $1995^{1}$. A partir de entonces su uso viene siendo reproducido por los especialistas de varios países, como interpretación del mismo. De acuerdo con Nunes Junior, citando a Vallinder, destaca que la judicialización de la política significa la transferencia de derechos de toma de decisiones de la legislatura, del gabinete o de la administración pública para los tribunales, así como, la difusión de los métodos de decisiones judiciales para ámbitos no jurídicos. "En resumen, podemos decir que la judicialización envuelve esencialmente transformar algo en proceso judicial" (TATE e VALLINDER,1995, p.13, apud JUNIOR, 2008:159) (traducción nuestra).

De esta forma, queda evidente que los autores enmarcan la judicialización en dos dimensiones, tanto en la política como en las relaciones sociales, que aparecen de forma amplia y dinámica en diversos contextos. Ambos autores Tate e Vallinder están de acuerdo en que es un fenómeno que remite a dos significados: uno se refiere al aumento del poder del Juez y del Tribunal, a través de la transferencia de las decisiones sobre políticas públicas del legislativo y del ejecutivo para el ámbito Judicial, generalmente asociado y fundamentado en la Constitución Política, lo que determina la defensa de la constitucionalidad o, en su defecto, la declaración de inconstitucionalidad de las leyes, actos y decisiones a ser tomadas o en vías de ejecución por los sectores políticos gubernamentales. Y, por otro lado, la diseminación de métodos judiciales de toma de decisiones por fuera del ámbito de los tribunales, reproducidos en la dinámica de las relaciones sociales.

Así efectivamente, crece la presencia del Poder Judicial, y la centralidad del juez, en el cotidiano de la vida social, a través de la interpretación jurídica que afecta, de diversas formas, sujetos individuales y colectivos de las clases sociales fundamentales que ingresan con litigios en el Poder Judicial o que son sometidos a procesos decisivos en los tribunales y salas especializadas. Lo que contribuye a romper con el carácter consuetudinario de regulaciones en espacios determinados por convenciones y tradiciones, incentivando la creciente presencia y respeto a las leyes y procedimientos burocráticos y administrativos, en consonancia con la defensa y garantía de derechos puestos en conflicto.

Por su vez, el autor Ran Hirschl, introdujo tres categorías, a partir de elementos que utiliza para analizar el concepto de judicialización de la política. Conforme palabras del mismo:

\footnotetext{
${ }^{1}$ Importante señalar que esta obra aún no ha sido traducida integralmente al español ni al portugués. Aqui consideramos algunos trechos citados en el artículo de Amandino Nunes Junior em 2008, que realizó una traducción própria.
} 
Comienzo por distinguir tres categorías amplias de judicialización: la diseminación de discursos, jergas, reglas y procedimientos jurídicos en la esfera política y en los fórums y procesos de elaboración de políticas públicas; la judicialización en la elaboración de políticas públicas por las formas "comunes" de control judicial, de constitucionalidad de leyes y actos de la administración pública; y la judicialización de la "política pura" - la transferencia, para los tribunales, de asuntos cuya naturaleza y significado son claramente políticos, incluyendo importantes debates sobre la legitimidad de regímenes e identidades colectivas que definen (y muchas veces dividen) comunidades enteras (HIRSCHL, 3:2009). (traducción nuestra)

De tal forma, se evidencia 1. la presencia de reglas y procedimientos jurídicos en los procesos de administrar y gestionar políticas públicas referidas a la presencia del Estado en la dinámica social, 2. el control judicial y la constitucionalidad de las leyes, y, 3. como novedad, lo que él llama de judicialización de la "política pura", la capacidad de los tribunales de incorporar o segregar diversidades culturales, aprobar regímenes, convocar plebiscitos, entre otros, de acuerdo con el ordenamiento constitucional vigente.

En suma, la judicialización puede ser interpretada, de acuerdo con estos autores, como un fenómeno en que predomina la transferencia frecuente de decisiones sobre políticas públicas para el ámbito Judicial, a partir del llamado control de constitucionalidad, que, de múltiples formas, condiciona las prácticas políticas de segmentos importantes de ciudadanos, actores individuales y colectivos. Así se configura, según ese enfoque, como un proceso sistémico, destinado a reproducir las condiciones objetivas de funcionalidad del orden constitucional establecido, a partir de la aceptación de reglas y controles que responsabilizan a las instituciones democráticas por el cumplimiento de sus funciones y quehaceres políticoadministrativos.

Consubstanciados en la perspectiva crítica de la totalidad, se evidencia el carácter pragmático en la utilidad socio-institucional de este concepto de judicialización, que está en marcha con la injerencia del Poder Judicial en el proceso político de la administración y control de las políticas públicas, sobre todo, a partir de las necesidades de mantenimiento y regulación de las condiciones históricas de reproducción de la sociedad capitalista. Lo que indica que la intervención del Poder Judicial es interinstitucional y social, fragmentada en las demandas que recibe y consolidando respuestas en función de las necesidades de mantenimiento del orden social vigente; lo que redunda en su carácter pragmático al momento de su intervención sistémica en el marco de las condiciones democráticas formalmente establecidas.

Así, la garantía del control constitucional y junto con ella la cuestión de la 
judicialización, no implican necesariamente, la ampliación y acceso a la justicia, una vez que esta se mantiene formal y abstracta para amplios sectores empobrecidos de las clases subalternas que, históricamente, fueron excluidos del sistema de garantía de derechos y de acceso al Poder Judicial. Lo que refleja el "movimiento que representa la continuidad de la utilización del derecho como hechizo, únicamente como una racionalización ideológica, que legitima la explotación capitalista" (SIERRA, 2011:257).

Por otro lado, si pensamos en la judicialización como una ampliación del acceso a la justicia, siendo percibida como una conquista de las clases subalternas en la defensa de la ciudadanía y de la democracia social, se vislumbraría un panorama que garante la efectiva participación y representación en el control de los derechos constitucionales. A este respecto, de acuerdo con Avritzer y Marona, la inclusión de amplios mecanismos de participación en los procesos de (re)constitucionalización de diversos países latinoamericanos, de que son ejemplos las enmiendas populares en Brasil y también las experiencias realizadas de plebiscitos y referendos realizados en Ecuador, Uruguay, Bolivia; además de otros mecanismos de ampliación de la representación y participación de actores sociales en el ámbito del control constitucional, indicarían un desafío actual frente a la judicialización de la política,

la ampliación de la dimensión representativa, con destaque para la actuación de los movimientos sociales, especialmente en el campo del debate constitucional, por un lado, y el del fortalecimiento de la representación política del Poder Judicial, por otro. (AVRITZER y MARONA, 2014:87).

Lo anterior, sin embargo, no implica necesariamente una creciente democratización de las instituciones político jurídicas del Estado, que como se sabe en las sociedades divididas en clases sociales tiene un límite determinado, por reglas de juego definidas en función de manutención del orden existente; una vez que, la dimensión representativa se refiere al hecho de que las decisiones colectivas no son tomadas por los propios sujetos directamente envueltos, sino por aquellas personas elegidas para esta finalidad, lo cual es determinado en función de la correlación de fuerzas sociales y políticas en disputa, las jerarquías institucionales, los intereses gubernamentales en juego, las movilizaciones de las clases y luchas sociales presentes, que contribuyen contradictoriamente con el proceso de ampliar la participación democrática y la garantía de reconocimiento de los derechos humanos para diferentes segmentos y grupos sociales. Aquí se entra en el ámbito de la lucha política, del carácter de clase de los conflictos sociales y del propio carácter de clase de los "guardianes e intérpretes" de la ley (jueces, magistrados y fiscales) que pueden y, de hecho, inciden en 
diferentes salidas frente al orden societario; no obstante, la mayor parte de las veces se posicionan ante los crecientes embates y conflictos sociales, en pro de la estabilidad jurídico / normativa de la institucionalidad vigente.

Esta dinámica se agrava en la actual coyuntura de reanudación de las medidas neoliberales y de financierización del capital, que ponen en el centro de las necesidades el "encanto de la mercancía". Las relaciones sociales se inclinan entre la vivencia de lazos conciliatorios posibles y la judicialización, por regla general, apela a esta constante necesidad, frente a su disolución encarnada en la barbarie, enfrentada cotidianamente por las clases e individuos sociales. Así, el clamor por la ley y el orden, respeto a las reglas del juego democrático, combinados con valores y principios autoritarios y prácticas conservadoras, configuran el núcleo dominante de intereses en el marco de la democracia burguesa que predomina en la actual cultura globalizada.

Según lo visto, la función del Poder Judicial se encuentra en medio de una tensión política e interventiva. Cabe resaltar que esta tensión esta puesta por la configuración de las determinaciones antes señaladas. No obstante, se amplia el reconocimiento del Poder Judicial, como instancia superior de carácter decisivo, en el control y defensa de la Constitución, para garantizar los derechos civiles, políticos, económicos, sociales y de minorías de los segmentos que tienen acceso a sus instancias. Lo que, por su vez, trae implicaciones socio-políticas de fondo, en el ámbito de las demandas puestas por la Seguridad Social, directamente relacionada a las políticas públicas que responden a las expresiones de la cuestión social. Así, como señala Sierra

El envolvimiento del Poder Judicial en la ejecución de las políticas públicas trajo una serie de implicaciones con el reconocimiento de los derechos de grupos sociales, por la opción de requerir judicialmente la consideración con la privación material a la que están sometidos. De ahí la tendencia a la invasión en el Poder Judicial por aquellos que, sin condiciones para recurrir a los servicios en el mercado, no consiguen garantizar sus derechos por el acceso a las políticas del gobierno (SIERRA, 2011: 257).

De esta manera, se destaca que la judicialización, al ser instrumentalizada por la población usuaria de los servicios públicos, abre camino a los programas para obtener financiamiento, sin embargo, con la implementación del neoliberalismo, la precariedad de los servicios, la escasez en la distribución de recursos presupuestarios y humanos, entre otros, impiden adecuadamente la garantía efectiva de las políticas públicas; lo que va a generar sobre las instituciones gestoras una presión política con relación a las demandas de los usuarios de los servicios prestados.

En este sentido, cabe analizar los efectos prácticos que pesan sobre los segmentos 
empobrecidos de las clases trabajadoras, de sus dificultades históricas de acceso a la justicia; y también, por la expansión de parámetros y procedimientos jurídicos en el control de actos de la administración pública que dicen respecto al financiamiento, elaboración y ejecución de políticas sociales; que como se sabe, son elaboradas para de alguna manera cubrir en parte las demandas de estos segmentos mayoritarios. Las implicaciones de la judicialización sobre las políticas sociales es el tema que trataremos a seguir.

\section{Judicialización de las políticas sociales: de qué se trata?}

En lo referente a la judicialización de las políticas sociales públicas podemos observar que existe una tensión latente entre el carácter de las demandas que, por regla general, pretenden establecer jurisdicción en beneficio de individuos, y los impactos de estas en la dinámica societaria, es decir, ¿de qué forma la intervención del Poder Judicial tiene como finalidad administrar, prevenir, remediar conflictos sociales y/o atenuar los resultados de la falta de efectividad y el acceso a las políticas públicas sociales? Como destacan Aguinsky y Alencastro:

Siempre que haya la falta de respeto a los derechos positivados, el Poder Judicial tiene, no sólo la atribución legal, sino la obligación ética de interpelar a la institución que sea, para que la ley se cumpla. Entendemos, sin embargo, que este ente estatal tendría una acción infinitamente más impactante y transformadora de las relaciones sociales, si actuase en la prevención de los conflictos sociales, preocupándose más por el interés colectivo que por el despacho de acciones recibidas, por regla general, de forma individual y por un grupo reducido o sector de la población que conoce sus derechos y posee condiciones de acceder al sistema de justicia (AGUINSKY Y ALENCASTRO, 2006: 22).

Lo que no debe ser tomado como un proceso de mano única, se sabe que en el proceso democrático la presencia activa de los llamados "grupos de presión", movimientos sociales y organizaciones de la clase obrera, así como medios de comunicación y de información de masas contribuyen como formadores de opinión, que abiertamente o entre bambalinas inciden en las decisiones judiciales; con lo que el proceso de judicialización de la política social se convierte en parte del conflicto de las luchas sociales, lo que demuestra los intereses en disputa entre las clases fundamentales y el papel mediador del poder del Estado.

En los Estados de Derecho (democrático-liberales) cabe la regulación de los conflictos sociales, controlar los movimientos de las clases subalternas que luchan abiertamente por el reconocimiento de los derechos humanos fundamentales, que procuran respuestas a sus necesidades y demandas sociales, aunque sean éstas ofrecidas en formatos focalizados y/o 
selectivos, incluso con soporte en el Mercado y participación privada. Por su vez, en el Welfare State se cumple un papel en perspectiva universal que anticipa la conflictividad social. Atravesado por políticas públicas sostenidas en criterios de universalidad, con baja o mínima participación de la iniciativa privada. De forma más amplia contribuye con la regulación social de la sociedad de clases. En fin, entre una y otra versión aparece la llamada judicialización de las políticas sociales que inciden en las expresiones de la cuestión social y las luchas de clases. Son las contradicciones propias del orden burgués, en que el Estado intermedia los procesos de las demandas de garantía de derechos humanos fundamentales y sociales.

De esa explanación, entre regulación social y judicialización, converge la relación entre la dimensión jurídica y la dimensión política sobre la cuestión social a través del tamiz de las políticas sociales, como respuestas del Estado a las demandas singulares en el marco de la defensa y garantía de la Seguridad Social. Netto llama la atención para la incorporación del carácter público de la cuestión social y de su direccionamiento, que refuerza la apariencia de la naturaleza privada de sus expresiones individuales.

En la escala en que se implementan medidas públicas para enfrentar las refracciones de la 'cuestión social', la permanencia de sus secuelas es dislocada para el espacio de la responsabilidad de los sujetos individuales que las experimentan (NETTO, 1997:26).

Esta determinación se constata en la sociedad capitalista que juega con la retórica del "derecho a tener derechos" (GUERRA, 2013), se refiere al carácter segregado y excluyente de la propia naturaleza de esta sociedad, que necesita la retórica de la promulgación de los derechos humanos y sociales para dar respuestas a los sujetos individuales como el punto más alto de la conquista de la civilidad burguesa, aunque sea ésta, formal y aparente. Con lo cual queda claro que en la práctica, los derechos reconocidos y las políticas sociales en esta sociedad se refractan en consecuencias individuales.

Por esa razón, los impactos mínimos en el interés colectivo, confirman la dificultad del Poder Judicial de tratar las expresiones de la cuestión social. Una vez que se centra en políticas sociales fragmentadas, dilaceradas por las medidas neoliberales implementadas en la mayor parte de los países de la región, que pasan distante de la perspectiva universalista. De esa forma sus respuestas son parciales, impregnadas de criterios selectivistas y focalizadas, adecuadas a los intereses del reducido segmento de la población que conoce sus derechos, lo que entra en conflicto con la finalidad de su discurso genérico sobre el acceso igualitario a la justicia por parte de todas las clases sociales. 
De manera general, en el ámbito del Poder Judicial como parte de la estrategia implementada en el proceso de judicialización de la política social, en que se destaca su atomización en respuesta a las demandas por derechos civiles, laborales, sociales, difusos, entre otros, la funcionalidad del Estado burgués replica la estrategia política de la fragmentación en la atención a las expresiones de la cuestión social ${ }^{2}$. La cuestión social aquí señalada la concebimos desde una perspectiva crítica

como expresión ampliada de la explotación del trabajo y de las desigualdades y luchas sociales que de ella derivan: lo contrario del desarrollo de las fuerzas productivas del trabajo social. Su producción / reproducción asume perfiles y expresiones históricamente particulares en el escenario contemporáneo latinoamericano (IAMAMOTO, 2007:162). (traducción nuestra).

En ese escenario, el Estado y sus instituciones que forman el Poder Judicial, el Poder Ejecutivo y el Legislativo pasa a tener un papel fundamental en la regulación de las relaciones sociales antagónicas propias de la sociedad burguesa.

En este sentido, es importante afirmar que la concepción de judicialización de las políticas sociales, derivadas de las expresiones de la cuestión social, la concebimos como: la referencia en procura de respuestas, en el ámbito del propio Estado por parte del Poder Judicial, a las demandas planteadas por los individuos sociales organizados, colectiva y/o singularmente, que ven diezmados sus derechos y garantía de acceso a la esfera de los servicios públicos disponibles por la política de seguridad social existente, en consonancia con las garantías constitucionales. Su mayor incidencia se da con el Poder Ejecutivo y con instituciones autónomas del Estado, donde actúan diversos profesionales que ejecutan de forma terminal las políticas sociales. Es la expresión concreta, en el cotidiano, de la fragilidad y precariedad de los establecimientos y servicios públicos, en los que los ciudadanos se insertan como usuarios de los mismos, y no logran acceder a los recursos y beneficios ofrecidos por la política social existente. De esta manera, como instancia superior, recurren al Poder Judicial para obtener garantía de su cumplimiento por parte de la institución ejecutora del Estado. Así, es evidente que

el programa para hacerle frente tiende a ser reducido a una gestión más humanizada y eficaz de los problemas sociales en la órbita del capital, bajo la batuta del capital financiero y de las políticas neoliberales (IAMAMOTO, 2007:162) (traducción nuestra).

\footnotetext{
${ }^{2}$ Estrategia, ya explicitada por Netto, en el seno de la intervención del Estado monopolista en la cuestión social: "En cuanto intervención del Estado burgués en el capitalismo monopolista, la política social debe constituirse necesariamente en políticas sociales: las secuelas de la "cuestión social" son recortadas como problemáticas particulares [...] y así enfrentadas" (Netto, 1997: 28).
} 
En suma, a partir de los argumentos levantados, se destaca lo que se refiere al proceso de judicialización de las políticas públicas sociales (salud, previsión, regímenes de jubilaciones y pensiones, asistencia, educación, entre otras) a través del control de constitucionalidad de las leyes y de los actos de la administración pública, en lo tocante a la transferencia de las decisiones del ámbito del Poder Ejecutivo para el del Poder Judicial; es decir, el camino de los recursos interpuestos por los usuarios en el Judicial con relación al incumplimiento de condiciones de acceso, permanencia, garantía y efectividad de los derechos sociales y humanos por parte del Poder Ejecutivo, en los diferentes niveles de organización de la administración pública.

La finalidad está centrada en obtener una decisión judicial que, imperativamente, someta a la administración pública y su ente enjuiciado a cumplir lo demandado. Lo que deja en evidencia la función de control judicial de actos administrativos, que se refieren a situaciones en las cuales los diversos entes públicos ejecutivos son demandados, singularmente, por individuos, grupos y colectivos organizados oriundos de la clase trabajadora mayoritariamente.

La judicialización muestra la contradicción y las dificultades de implementación y reproducción de la política social establecida, una vez que explicita la inadecuada distribución de fondos presupuestarios, la insuficiencia de recursos humanos y las cada vez más precarias condiciones de trabajo en los establecimientos públicos, así como la corrupción sistémica entre outros aspectos. Lo que lleva a configurar una judicialización de las políticas sociales con respuestas precarias, fragmentadas y selectivas, en que prevalece el discurso genérico en defensa de la garantía y protección de los derechos humanos fundamentales.

\section{Subsidios para el debate}

La configuración del discurso sobre la judicialización en su interrelación con la política social, trae a la superficie la problemática contradictoria de la justicia, de la desigualdad social, económica, política y cultural, condensada en la "cuestión social", que se concibe en el plano de la sociedad del capital en su forma real: la ciudadanía burguesa (MARSHALL, 1967) ${ }^{3}$. En esta constatación, cabe destacar, que la intervención del Estado de Derecho, sobre las refracciones de la cuestión social, mantiene el carácter fragmentador y de atención

\footnotetext{
${ }^{3}$ Ciudadanía manifiesta en el contrato social entre sujetos individuales, respetando reglas del juego democrático burgués. Estamos hablando de la conformación de la ciudadanía liberal burgués, formal - abstracta, que defiende el discurso de derechos civiles, políticos, sociales y culturales para la ciudadanía en general sin distinciones por inserción de clase, raza, género y etnia.
} 
parcializada sobre las demandas planteadas por los usuarios y usuarias de las clases subalternas, como ya se ha visto.

De esta manera, el Poder Judicial, a través de la judicialización de la política social y de las relaciones sociales, reproduce ese carácter cuando interviene en las refracciones de la cuestión social, con el propósito de controlar judicialmente y administrar la política social pública. Su intervención se limita a actuar junto con los otros poderes en la perspectiva de "mejorar el proceso democrático existente" (BARBOZA e KOZICKI, 2012:79). Pero, el fenómeno de la judicialización, de hecho, no administra ni controla la propia cuestión social; una vez que, explicitaría la esencia de la explotación y de la injusticia social en el orden burgués. Así se entiende como equivocado el uso de la acepción “judicialización de la cuestión social".

Y no puede ser de otro modo: tomar la "cuestión social" como problemática configuradora de una totalidad procesal específica es remitirla concretamente a la relación capital / trabajo - lo que significa, en su límite, poner en jaque al orden burgués. [...] Así, la "cuestión social" es atacada en sus refracciones, en sus secuelas comprendidas como problemáticas cuya naturaleza totalizante, si asumida consecuentemente, impediría la intervención (NETTO, 1996: 28).

En este marco, el rasgo característico de las decisiones judiciales es tratar de hacer justicia entre los desiguales, intenta establecer condiciones de reconstitución del tejido social a través de la regulación de la participación de las clases antagónicas institucionalmente, contando con la trayectoria asimétrica, a veces, no reconocida e ilegitimada de las clases subalternas. La gran responsabilidad de este proceso en el caso que nos ocupa, está dada a la política pública social, que en su doble estrategia atende tanto a las "necesidades del capital como del trabajo, ya que para muchos se trata de una cuestión de sobrevivencia" (Behring, 1998:175). De ahí la importancia que la política social sea reconocida por el Estado en una perspectiva de acceso universal para quien de ella necesite, rompiendo con visiones restrictas.

Sin embargo, se debe notar que si las políticas sociales no tienen el poder de erradicar la cuestión social, también es cierto que ellas posibilitan derechos sociales, frutos de largo proceso de luchas históricas de los trabajadores por su reconocimiento político. Y a ellas también se alían iniciativas del bloque dominante en la concesión de derechos, anticipándose a las reivindicaciones oriundas de diferentes segmentos sociales, según estrategias de desmovilización de las luchas sociales. El campo de las políticas públicas y de los derechos sociales es también una arena de acumulación de fuerzas políticas de luchas en torno a proyectos para la sociedad en el enfrentamiento de las desigualdades condensadas en la cuestión social (IAMAMOTO, 2007: 275). (traducción nuestra).

En ese proceso se entiende que la judicialización de las políticas sociales, tal y como 
concebida y efectuada, no propicia una superación de los problemas de fondo que pretende afrontar. Hay que considerar que el fenómeno se presenta ante necesidades insatisfechas en la base material de la propia sociedad capitalista que las produce; que requiere modificaciones profundas en el modo de producir los bienes y recursos, la riqueza social, y sus maneras de distribución y apropiación por parte de los individuos y colectivos sociales de las clases subalternas.

\section{Consideraciones finales}

Como visto el concepto de judicialización diseminado en la actualidad, de acuerdo con el análisis realizado, se estructura en el marco de la institucionalidad vigente, como parte de una perspectiva pragmática que intenta organizar y orientar las demandas que ingresan al Poder Judicial, visionando el control de la constitucionalidad y la adecuación de la administración pública a las condiciones mínimas de garantía de los derechos pleiteados por los diversos segmentos de las clases sociales fundamentales, lo que conlleva una serie de mecanismos y de discursos que justifican las medidas tomadas, en su amplia mayoría reproductoras y legitimadoras del orden social existente. En este sentido, la judicialización, lejos de configurar un fenómeno aislado, atraviesa la dinámica de la vida social y cumple una función, en el marco del Estado contemporáneo, de servir a los intereses de mantener la regulación jurídica, social y económica entre las clases antagónicas en conflicto.

De acuerdo con el enfoque de la totalidad asumido, se evidencia que la judicialización de las políticas sociales, por sus características históricamente determinadas, se configura a partir de condiciones objetivas, cuando el Poder Judicial es accionado para fallar y sancionar el cumplimiento de derechos sociales por parte de la administración pública, de instituciones autónomas y del Poder Ejecutivo que lidian con la implementación de políticas sociales. De esta forma, se demuestra como la judicialización de las políticas sociales se convierte en una de las estrategias del Estado de Derecho para enfrentar y dar respuestas a las expresiones de la cuestión social contemporánea.

\section{Referencias bibliográficas}

AVRITZER, Leonardo e MARONA, Marjorie. Judicialización da política no Brasil: ver além do constitucionalismo liberal para ver melhor. in Revista Brasileira de Ciência Política no.15 Brasília Sep./Dec. 2014. Disponible en: http://dx.doi.org/10.1590/0103-335220141504. Acceso en 12 de noviembre 2016. 
AGUINSKY, B.G. y ALENCASTRO, E.H. Judicialização da questão social: rebatimento no processo de trabalho dos assistentes sociais no Poder Judicial. Revista Katálysis, Florianópolis, jan./jun. 2006. Acceso en 08 de noviembre de 2016.

BARBOZA, E. M.Q e KOZICKI, K. Judicialización da política e controle judicial de políticas públicas. Revista DireitoGV, São Paulo N. 15 p. 059-086, jan/-jun. 2012. Disponible en http://www.scielo.br/pdf/rdgv/v8n1/v8n1a03.pdf. Acceso en 10 de noviembre 2016.

BEHRING, E. R. Política social no capitalismo tardio. Sao Paulo: Editora Cortez, 1998.

GUERRA, Y. Direitos Sociais e Sociedade de Classes: o Discurso do direito a ter direitos. In: Ética e direitos: ensaios críticos. $4^{\mathrm{a}}$ ed. Coletãnea Nova de Serviço Social. Rio de Janeiro, Lumen Juris, p.31-54. 2013.

HIRSCHL, Ran. O novo constitucionalismo e a judicialización da política pura no mundo. Revista de derecho Administrativo, Rio de Janeiro, v. 251, p. 139-178, mai. 2009. ISSN 2238-5177. Disponible en: http://bibliotecadigital.fgv.br/ojs/index.php/rda/article/view/ 7533.://dx.doi.org/10.12660/rda.v251.2009.7533. Acesso em: 06 de abril 2017

IAMAMOTO, M. V. Serviço Social en tempo de capital fetiche: capital financeiro, trabalho e cuestión social. São Paulo: Cortez, 2007.

JUNIOR, A. T. A Constitución de 1988 e a judicialización da política no Brasil. Revista de Informação Legislativa. Brasilia. a. 45. n. 178, p. 157-167. abril/junho. 2008. Disponible en: http://www2.senado.leg.br/bdsf/bitstream/handle/id/496910/RIL178.pdf. Acceso en 02 noviembre de 2016.

MARSHAL, T. H. Cidadania, classe social e status. Rio de Janeiro: Editora Zahar, 1967.

NETTO, J. P. Capitalismo monopolista y Servicio Social. São Paulo: Editora Cortez, 1997.

SIERRA, V. M. A judicialización da política no Brasil e a atuação do assistente social na justiça. Revista Katálysis. Florianópolis. Vol 14. No2. p. 256-264, jul-dez 2011.

TATE, C. N. y VALLINDER, T. (organizadores). The global expansion of judicial power. New York University 1995. 